\title{
A!
}

This is an electronic reprint of the original article.

This reprint may differ from the original in pagination and typographic detail.

Shevchenko, A.; Grahn, P.; Kaivola, M.

\section{Internally twisted spatially dispersive optical metamaterials}

Published in:

Journal of Nanophotonics

DOI:

10.1117/1.JNP.8.083074

Published: 01/01/2014

Document Version

Publisher's PDF, also known as Version of record

Please cite the original version:

Shevchenko, A., Grahn, P., \& Kaivola, M. (2014). Internally twisted spatially dispersive optical metamaterials. Journal of Nanophotonics, 8(1), 1-12. [083074]. https://doi.org/10.1117/1.JNP.8.083074

This material is protected by copyright and other intellectual property rights, and duplication or sale of all or part of any of the repository collections is not permitted, except that material may be duplicated by you for your research use or educational purposes in electronic or print form. You must obtain permission for any other use. Electronic or print copies may not be offered, whether for sale or otherwise to anyone who is not an authorised user. 


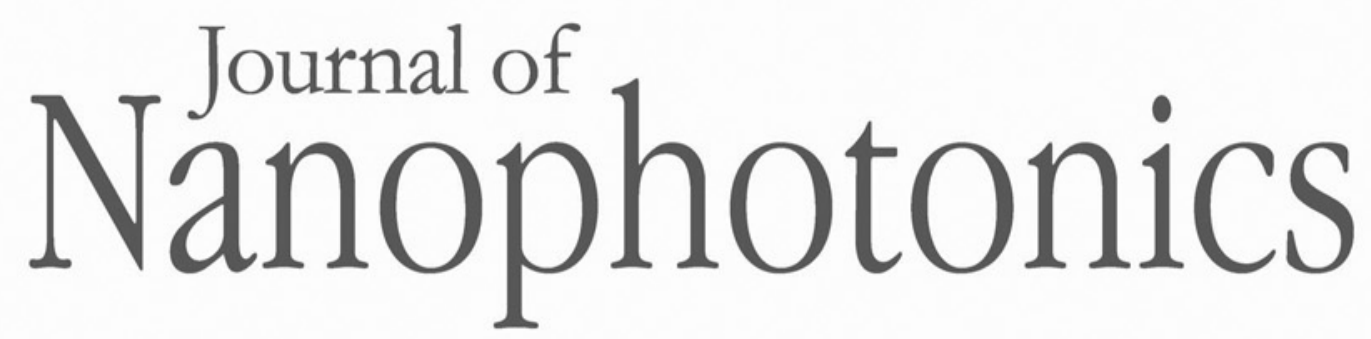

Nanophotonics.SPIEDigitalLibrary.org

\section{Internally twisted spatially dispersive optical metamaterials}

Andriy Shevchenko

Patrick Grahn

Matti Kaivola 


\title{
Internally twisted spatially dispersive optical metamaterials
}

\author{
Andriy Shevchenko, , Patrick Grahn, and Matti Kaivola \\ Aalto University, Department of Applied Physics, P.O. Box 13500, FI-00076 Aalto, Finland
}

\begin{abstract}
We introduce and theoretically describe internally twisted optical metamaterials, in which the metamolecules can have an arbitrary, but common, orientation in the unit cells. The molecules, and consequently the material, are not chiral, but they are allowed to be noncentrosymmetric. While such internally twisted crystalline structures are difficult to find in natural materials, metamaterials of this type can be designed and fabricated at will. Here, we present a theoretical method that enables a detailed analysis of such metamaterials. The method establishes a connection between the optical properties of a metamaterial and the plane-wave optical response of a single two-dimensional array of metamolecules. In the theory, the effective wave parameters, such as the refractive index and wave impedance, are retrieved. Using the model, we show that these parameters can dramatically depend on the wave propagation direction and metamolecular orientation, which can be used, along with optical anisotropy, to efficiently adjust and control the plane-wave content of optical beams. (C) 2014 Society of Photo-Optical Instrumentation Engineers (SPIE) [DOI: 10.1117/1.JNP8583074]
\end{abstract}

Keywords: metamolecular orientation; propagationally anisotropic metamaterials; noncentrosymmetric scatterers; spatial dispersion; refractive index; impedance.

Paper 14074P received Jul. 11, 2014; revised manuscript received Sep. 24, 2014; accepted for publication Sep. 26, 2014; published online Oct. 27, 2014.

\section{Introduction}

Modern computation and nanofabrication technologies make it possible to design and fabricate a wide range of artificial optical materials with the aim of manipulating light in desired ways. Based on the size of the unit cells relative to the light wavelength, these materials can be conditionally divided into three categories. Photonic crystals compose one of them. They are periodic structures characterized by highly symmetric constituents, such as spheres and holes, with the unit-cell size being on the order of $\lambda / 2, \lambda$ being the wavelength of light. These materials exhibit photonic band gaps used to confine and guide optical waves. Photonic crystals are often characterized by Bloch modes and the corresponding dispersion diagrams, such as isofrequency surfaces and contours. Light propagation in a photonic crystal is governed by diffraction and depends on the propagation direction. The second category includes materials with unit cells that are several orders of magnitude smaller than $\lambda$. The optical properties of such a material depend only on the local strength and orientation of the electric field component of light. These materials can be described in terms of an electric permittivity tensor or refractive index ellipsoid using effective medium theories. ${ }^{\mathrm{Q}}$ They are not optically magnetic and their properties are independent of the light propagation direction. We, therefore, call these materials propagationally isotropic metamaterials. The third category of artificial materials, which is the type considered in this work, consists of those that fit in the range between the mentioned photonic crystals and the propagationally isotropic metamaterials. In these materials, the metamolecules have designed nontrivial shapes and the unit-cell size is shorter than $\lambda / 2$, but not by much. As a result, while no diffraction orders can appear in any direction, the material's optical response is influ-

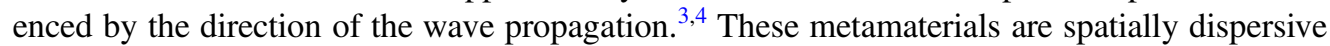
and we, therefore, call them propagationally anisotropic metamaterials. Their effective refractive

*Address all correspondence to: Andriy Shevchenko, E-mail: andriy.shevchenko@aalto.fi

0091-3286/2014/\$25.00 (C) 2014 SPIE 
index and wave impedance are well-defined. Furthermore, in such metamaterials, the moments of higher-order multipoles, such as electric quadrupoles or magnetic dipoles, are not negligible. Consequently, the effective wave impedance is no longer determined by the refractive index. Therefore, the characterization of these metamaterials requires both the effective refractive index and wave impedance along with their dependence on the propagation direction.

Using the mentioned propagationally anisotropic metamaterials, one can achieve many extraordinary optical phenomena similar to those observed in photonic crystals, but without diffraction effects and with adjustable wave impedance. These phenomena include the superprism

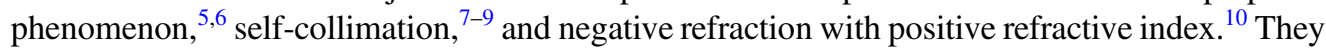
can appear due to noncircular isofrequency contours caused by spatial dispersion. The spatial dispersion, in turn, can be enhanced by breaking the symmetry of the material. Previously, we had designed a material in which the metamolecules are not centrosymmetric, which makes the material bifacial $\square$ In this work, we consider noncentrosymmetric metamolecules which are tilted with respect to the crystal lattice axes. This further enhances the propagational anisotropy of the metamaterial. Since the metamolecules no longer share their symmetry axis with the lattice, we refer to the material as internally twisted.

The optical properties of an arbitrary metamaterial can be fully characterized in terms of the metamaterial's response to optical plane waves of different frequencies, polarizations, and propagation directions. For crystalline metamaterials, the plane-wave response can be efficiently evaluated using a recently proposed interferometric approach. ${ }^{2}$ Within this approach, the metamaterial crystal is divided into planar arrays of metamolecules, as shown in Fig. [. The overall interaction of light with each array is determined by plane-wave transmission and reflection coefficients that, in general, depend on the light frequency, polarization and propagation direction. The interaction picture is obtained by considering the multiple reflections and transmissions of light by the consecutive metamolecular layers, as shown in Fig. 1.

To explain the approach in more detail, we consider a plane wave propagating at an angle $\theta$ with respect to the $z$ axis (see Fig. (1). The crystal planes are periodically separated by the lattice constant $\Lambda_{z}$. The finite reflection coefficient of the sheets, with which we replace the horizontal arrays of molecules, produces a second wave that propagates at an angle of $180^{\circ}-\theta$ with respect to the $z$ axis. In general, the transmission and reflection coefficients $\tau_{2}$ and $\rho_{2}$ experienced by this reflected wave differ from $\tau_{1}$ and $\rho_{1}$ of the original wave. Only for propagation along the $z$ axis does the equality $\tau_{1}=\tau_{2}$ necessarily hold. Here, we consider arbitrary propagation directions

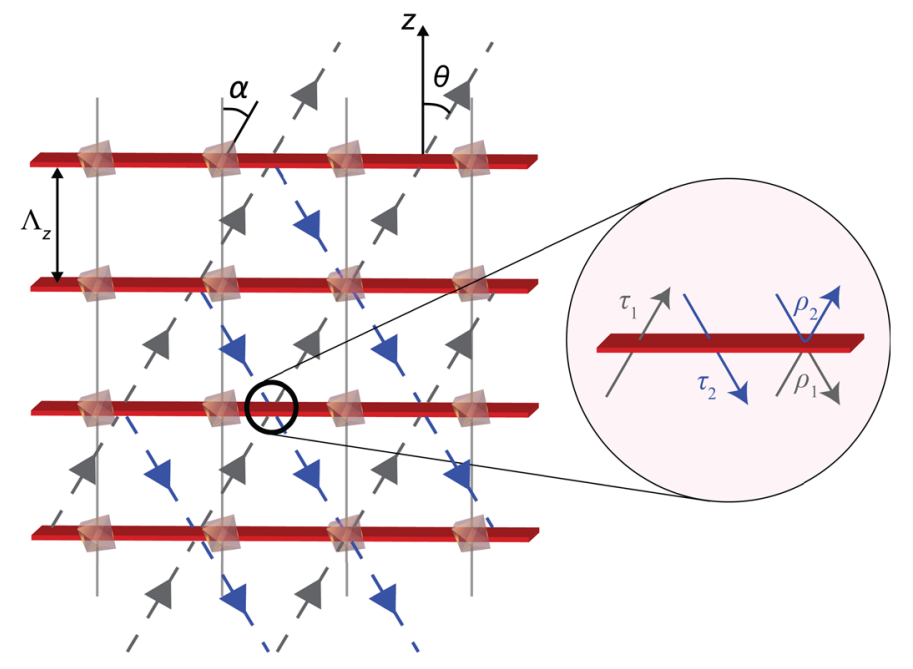

Fig. 1 Interferometric description of optical metamaterials. Each plane of nanoscatterers is treated as an infinitesimally thin sheet with plane-wave transmission and reflection coefficients $\tau$ and $\rho$. A plane wave propagating upwards along the gray arrows at an angle $\theta$ with respect to the $z$ axis is reflected by the sheets, creating a second wave propagating downwards along the blue arrows. The coefficients $\tau$ and $\rho$ of this wave can differ from those of the incident wave, i.e., $\tau_{1} \neq \tau_{2}$ and $\rho_{1} \neq \rho_{2}$. These two waves are coupled to each other by the multiple reflections at the crystal planes. 
and polarizations, for which $\tau_{1} \neq \tau_{2}$ and $\rho_{1} \neq \rho_{2}$. Our consideration also includes metamaterials that are not symmetric with respect to the $z$ axis. The metamolecules are not chiral, but are twisted (their symmetry axis is tilted by an angle $\alpha$ with respect to $z$ ), resulting in the inequality $\tau_{1} \neq \tau_{2}$. A theory suitable for such metamaterials has not been previously introduced. Hence, we do this in the following section. Then, in Sec. 3, we apply the theory to a particular, but generic example of a dimer metamaterial and evaluate the influence of the twist angle and the light incidence angle on the material's optical response. We also address the possibility of using spatial dispersion for control of propagational characteristics of optical beams. In Sec. 匇, we summarize our results.

\section{Theoretical Description}

The model presented in this section is based on our previous theoretical developments that concern bifacial metamaterials (see Refs. 11] and 12). In the following, the theory is generalized to also include internally twisted metamaterials. Independent of the presence of a twist, the recursive relation that connects the fields between adjacent crystal planes of a metamaterial can be written as [see Eq. (3) in Ref. [12]

$$
\beta U_{j+1}+U_{j-1}-\alpha U_{j}=0,
$$

where $U_{j}$ denotes the amplitude of the forward-propagating plane wave after the $j$ 'th plane of metamolecules. For a general material that can be both twisted and noncentrosymmetric, the parameters $\alpha$ and $\beta$ are related to two phase-shifted transmission coefficients, $f_{1}=$ $\tau_{1} \exp \left(\mathrm{i} k_{z} \Lambda_{z}\right)$ and $f_{2}=\tau_{2} \exp \left(\mathrm{i} k_{z} \Lambda_{z}\right)$, and two phase-shifted reflection coefficients, $g_{1}=$ $\rho_{1} \exp \left(\mathrm{i} k_{z} \Lambda_{z}\right)$ and $g_{2}=\rho_{2} \exp \left(\mathrm{i} k_{z} \Lambda_{z}\right)$, by

$$
\begin{gathered}
\alpha=f_{2}+f_{1}^{-1}\left(1-g_{1} g_{2}\right), \\
\beta=f_{2} / f_{1} .
\end{gathered}
$$

Here, $k_{z}$ denotes the $z$ component of the wave vector in the host medium that surrounds the scatterers. If the material was not internally twisted, the transmission coefficients $\tau_{1}$ and $\tau_{2}$ would be equal.

Next, we denote the wave number in the effective medium by $\gamma$ and the $z$ component of the corresponding wave vector by $\gamma_{z}$, and require that translation by one unit cell in the effective medium satisfies the periodicity condition

$$
U_{j}=U_{j-1} \exp \left(\mathrm{i} \gamma_{z} \Lambda_{z}\right)
$$

Inserting Eq. (ד) into Eq. (11) allows us to solve for

$$
\gamma_{z} \Lambda_{z}=-\mathrm{i} \ln \left[\frac{\alpha}{2 \beta} \pm\left(\frac{\alpha^{2}}{4 \beta^{2}}-\frac{1}{\beta}\right)^{1 / 2}\right]+2 \pi m,
$$

where $m$ is an integer. At an interface between the metamaterial and the host medium, the wave vector components that are tangential to the interface are preserved, i.e., $\gamma_{x}=k_{x}$ and $\gamma_{y}=k_{y}$. Thus, the effective refractive index $n_{\text {eff }}$ can straightforwardly be calculated from

$$
\gamma^{2}=n_{\mathrm{eff}}^{2} k_{0}^{2}=k_{x}^{2}+k_{y}^{2}+\gamma_{z}^{2},
$$

where $k_{0}$ denotes the wave number in vacuum. Together, Eqs. (5) and (6) enable the determination of the effective refractive index from the transmission and reflection coefficients of a single planar array of nanoscatterers. This approach considers the evanescent-wave coupling between the metamolecular layers as negligible, which is justified for metamaterials with subwavelength unit cells and well-separated metamolecules. [1. [-

In order to obtain the effective wave impedance of a metamaterial, one must average the electric and magnetic fields inside the material. According to Ref. 12, the effective wave impedance can be expressed as 


$$
Z_{\text {eff }}=Z\left(\frac{k \gamma_{z}}{k_{z} \gamma}\right)^{p} \frac{\left\langle U_{j} \exp \left(\mathrm{i} k_{z} z\right)+U_{j}^{\prime} \exp \left(-\mathrm{i} k_{z} z\right)\right\rangle}{\left\langle U_{j} \exp \left(\mathrm{i} k_{z} z\right)-U_{j}^{\prime} \exp \left(-\mathrm{i} k_{z} z\right)\right\rangle}
$$

where $Z$ is the impedance of the host medium and $p$ is equal to +1 and -1 for TE- and TMpolarized light, respectively. The factor $\left(k \gamma_{z} / k_{z} \gamma\right)^{p}$ accounts for the fact that the propagation direction of the effective wave differs from that in the host medium. The variable $U_{j}^{\prime}$ denotes

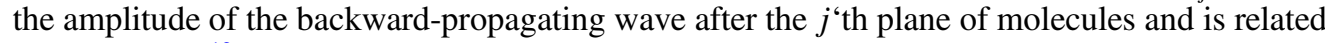
to $U_{j}$ through

$$
U_{j}=f_{1} U_{j-1}+g_{2} U_{j}^{\prime}
$$

Performing the spatial averaging over $z$ in Eq. (7) and using Eq. (8), we obtain the following expression for the effective wave impedance:

$$
Z_{\text {eff }}=Z\left(\frac{k \gamma_{z}}{k_{z} \gamma}\right)^{p} \frac{g_{2}+\left[1-f_{1} \exp \left(-\mathrm{i} \gamma_{z} \Lambda_{z}\right)\right]}{g_{2}-\left[1-f_{1} \exp \left(-\mathrm{i} \gamma_{z} \Lambda_{z}\right)\right]}
$$

The effective wave parameters $n_{\text {eff }}$ and $Z_{\text {eff }}$, along with their dependence on the wave frequency, propagation direction, and polarization, form a complete macroscopic description of a twisted crystalline metamaterial. In order to calculate the reflection, transmission, and refraction at a metamaterial boundary, one needs to know the Fresnel coefficients. In Ref. 11, these coefficients are generalized for uniaxial bifacial metamaterials. In order to allow for a wave undergoing internal reflection from the material's boundary to have different refractive indices before and after the reflection, we further generalize these coefficients and cover for internally twisted metamaterials as well.

Consider a boundary at $z=0$ between two materials of which either one can be a twisted metamaterial. Suppose that a plane wave is incident from the first material with a wave vector $\left(\gamma_{x}, \gamma_{y}, \gamma_{z, \mathrm{i}}\right)$, wave number $\gamma_{\mathrm{i}}$, and wave impedance $Z_{\mathrm{i}}$. In general, we can express the characteristics of the wave reflected by the interface as $\left(\gamma_{x}, \gamma_{y},-\gamma_{z, \mathrm{r}}\right)$ and $Z_{\mathrm{r}}$. Although one would obtain $\gamma_{z, \mathrm{r}}=\gamma_{z, \mathrm{i}}, \gamma_{\mathrm{r}}=\gamma_{\mathrm{i}}$, and $Z_{\mathrm{r}}=Z_{\mathrm{i}}$ for conventional materials, these equalities will not necessarily hold for metamaterials. For example, for uniaxial bifacial metamaterials, the impedances can be different $\square$ For the internally twisted metamaterials, the refractive indices and, therefore, the wave vectors can have different magnitudes. For the wave transmitted by the interface, we define the characteristics $\left(\gamma_{x}, \gamma_{y}, \gamma_{z, \mathrm{t}}\right), \gamma_{\mathrm{t}}$, and $Z_{\mathrm{t}}$. Applying the electromagnetic boundary conditions, we obtain the generalized Fresnel transmission and reflection coefficients, $\tau_{\mathrm{F}}$ and $\rho_{\mathrm{F}}$, for internally twisted metamaterials to be

$$
\begin{gathered}
\tau_{\mathrm{F}}=\frac{\gamma_{z, \mathrm{i}} /\left(\gamma_{\mathrm{i}} Z_{\mathrm{i}}^{\sigma}\right)+\gamma_{z, \mathrm{r}} /\left(\gamma_{\mathrm{r}} Z_{\mathrm{r}}^{\sigma}\right)}{\gamma_{z, \mathrm{r}} /\left(\gamma_{\mathrm{r}} Z_{\mathrm{r}}^{\sigma}\right)+\gamma_{z, \mathrm{t}} /\left(\gamma_{\mathrm{t}} Z_{\mathrm{t}}^{\sigma}\right)}\left(\frac{Z_{\mathrm{i}}}{Z_{\mathrm{t}}}\right)^{(\sigma-1) / 2}, \\
\rho_{\mathrm{F}}=\frac{\gamma_{z, \mathrm{i}} /\left(\gamma_{\mathrm{i}} Z_{\mathrm{i}}^{\sigma}\right)-\gamma_{z, \mathrm{t}} /\left(\gamma_{\mathrm{t}} Z_{\mathrm{t}}^{\sigma}\right)}{\gamma_{z, \mathrm{r}} /\left(\gamma_{\mathrm{r}} Z_{\mathrm{r}}^{\sigma}\right)+\gamma_{z, \mathrm{t}} /\left(\gamma_{\mathrm{t}} Z_{\mathrm{t}}^{\sigma}\right)}\left(-\frac{Z_{\mathrm{i}}}{Z_{\mathrm{r}}}\right)^{(\sigma-1) / 2},
\end{gathered}
$$

where $\sigma= \pm 1$ for the TE and TM polarization, respectively. Here, a positive real-valued $\rho_{\mathrm{F}}^{\mathrm{TM}}$ indicates that the incident and reflected waves have their tangential components of the electric field in phase. We note that, since the material is not chiral, the polarizations of the TE and TM waves are preserved when the waves interact with the material. The derived Fresnel coefficients can be used to calculate, e.g., the transmission and reflection coefficients of metamaterial slabs of an arbitrary thickness for any propagation angle and polarization of the incident wave in terms of the refractive index and impedance evaluated for this wave.

\section{Metal Dimer Metamaterial}

In this section, we consider an example of a metamaterial that is composed of asymmetric paired silver discs in a cubic lattice with a $120 \mathrm{~nm}$ lattice constant [see Fig. 2(a)]. These metamolecules are found to exhibit significant magnetic dipole and electric quadrupole polarizabilities ${ }^{15}$ and 
(a)

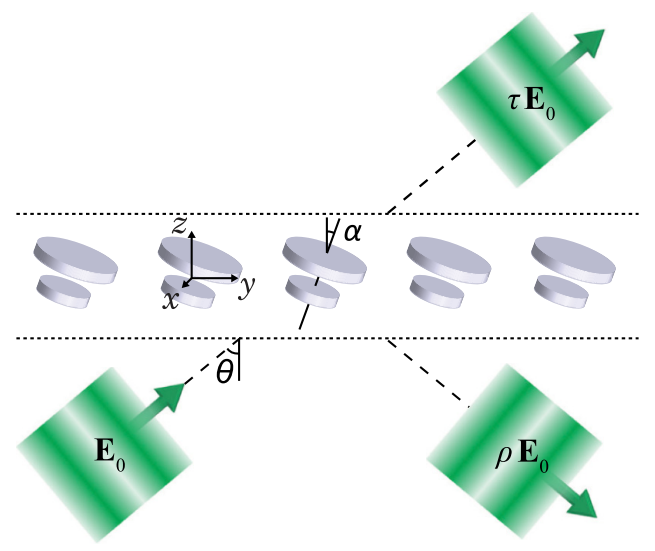

(b)

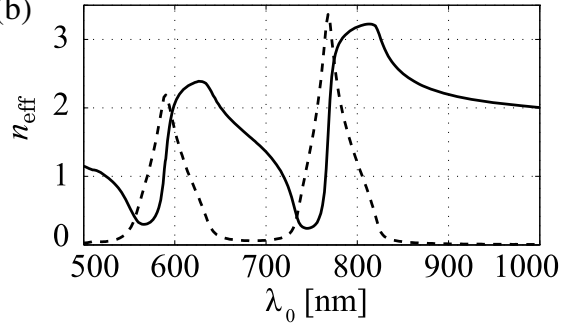

(c)

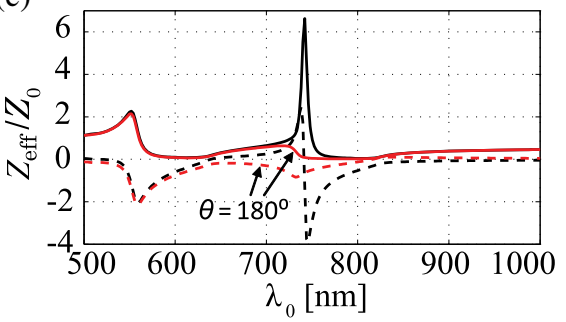

Fig. 2 (a) Transmission and reflection of a plane wave by a single plane of metal nanodimers composing the metamaterial crystal. In the spectra, the black lines show the calculated effective (b) refractive index and (c) wave impedance (normalized to that of vacuum) of the metamaterial for waves propagating at $\theta=0$, when $\alpha=0$. The wave impedance for a counter-propagating wave $(\theta=180 \mathrm{deg})$ is shown with red lines. The real and imaginary parts are shown by solid and dashed lines, respectively.

spatial dispersion ${ }^{\mathbb{Q}_{\text {in }}}$ the visible spectral range. Using the electromagnetic multipole theory, $\mathbb{1}_{\text {it }}$ can be verified that the dominant higher-order multipole excitation in the particles is composed of linear currents in the two discs oscillating out-of-phase with respect to each other. We choose the larger disc to have a radius of $40 \mathrm{~nm}$ and the smaller one $25 \mathrm{~nm}$. Both discs have a thickness of $10 \mathrm{~nm}$ and they are separated by a surface-to-surface distance of $20 \mathrm{~nm}$. The dimer axis is allowed to be tilted by an angle $\alpha$ in the $y z$-plane. The angle of incidence of a plane wave is denoted by $\theta$, such that the plane of incidence coincides with the $y z$-plane. Also, when $\theta$ is equal to $\alpha$, the wave is incident from the smaller-disc side. A dielectric host medium of refractive index 1.5 is assumed throughout this work.

In order to calculate the effective wave parameters of the metamaterial, we first numerically calculate the transmission and reflection coefficients of a single planar array of the dimers. The numerical calculations are performed using the computer software COMSOL Multiphysics and the values for the refractive index of silver are taken from Ref. 16. For each incidence angle $\theta$, two calculations are needed: one for a wave incident at $\theta$ (giving $\tau_{1}$ and $\rho_{1}$ ) and one for a wave incident at an angle of $180 \mathrm{deg}-\theta$ (giving $\tau_{2}$ and $\rho_{2}$ ). Then, using Eqs. (5) and (9), we calculate the effective wave parameters for the metamaterial crystal. Since the material is both spatially dispersive and anisotropic, the retrieved parameters depend on the polarization and propagation direction of light. In Figs. 2(b) and 2(c), we show the spectra of the wave parameters retrieved for the simplest case, where $\theta=0$ and $\alpha=0$. In the refractive index spectrum, one can distinguish the dipole resonances of the two discs composing the dimer. For $\alpha=0$, we can have $\rho_{1} \neq \rho_{2}$, but the equality $\tau_{1}=\tau_{2}$ must be the case for all choices of $\theta$. Indeed, for a counterpropagating wave $(\theta=180 \mathrm{deg})$, the refractive index is the same as for the original wave, but the impedance shown by the red lines in Fig. 2(c) is different. Thus, this metamaterial is optically bifacial.

The metamaterial becomes internally twisted if the dimers are tilted by an angle $\alpha \neq 0$. As an example, we choose $\alpha=45 \mathrm{deg}$. By calculating the transmission and reflection coefficients for $\theta=45 \operatorname{deg}$ and $\theta=135 \mathrm{deg}$ - such that the wave propagates along the symmetry axis of the dimers and, in the second case, perpendicular to it-we obtain the effective parameters $n_{\text {eff }}$ and $Z_{\text {eff }}$ for these waves. These parameters are shown in Fig. B for TE-polarized plane waves propagating in the directions of $\theta=45 \mathrm{deg}$ and $\theta=135 \mathrm{deg}$. The TE polarization is chosen to exclude the influence of optical anisotropy on the results obtained for different propagation 
(a)

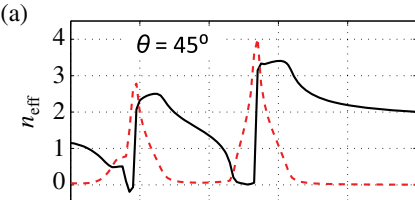

(c)

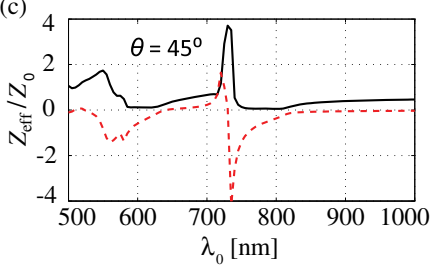

(b)

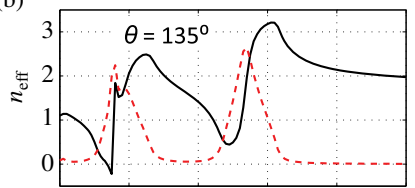

(d)

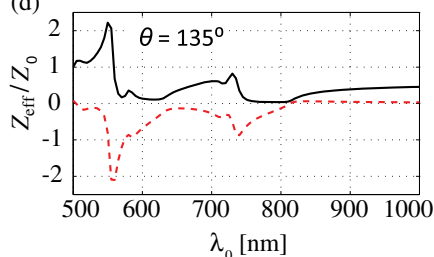

Fig. 3 Effective wave parameters for a metamaterial with the dimers tilted by $\alpha=45$ deg. The waves are TE-polarized. The effective refractive index and wave impedance are shown for waves propagating at [(a) and (c)] $\theta=45 \mathrm{deg}$ and [(b) and (d)] $\theta=135 \mathrm{deg}$. The real and imaginary parts are shown by solid and dashed lines, respectively.

angles of the wave. We notice that the two waves experience different refractive indices and different wave impedances. At certain wavelengths, blue detuned from the resonances, the real part of the refractive index takes values close to zero and even becomes negative (compare to Fig. (2). This feature shows that the effect of a twist can be remarkable.

\subsection{Verification of the Approach}

In order for a metamaterial to be treatable as a homogeneous material, it is necessary that the calculated wave parameters do not depend on the size of the crystal. This is equivalent to the requirement that the evanescent-wave coupling between adjacent crystal planes is weak. [2] According to the practical criterion proposed in Ref. 12 for noble-metal metamolecules, the gap $d$ between the particles in the $z$-direction must be larger than $\Lambda_{\max } / 2$, where $\Lambda_{\max }$ is the largest lattice constant of the crystal plane. For the considered dimer metamaterial, we have $d \approx 80 \mathrm{~nm}$ and $\Lambda_{\max } / 2=60 \mathrm{~nm}$, so that the metamaterial is expected to be homogenizable. We notice, however, that if the metamolecules are off-resonant or made of a dielectric material, this requirement is relaxed, since the evanescent fields produced by them are weak.

In order to verify the correctness of our semianalytical calculation approach, we first apply COMSOL to directly compute the transmission and reflection spectra for five molecular layers of a metamaterial with $\alpha=45 \mathrm{deg}$ at $\theta=45 \mathrm{deg}$. The interaction geometry is depicted in Fig. 4(a). The obtained intensity transmission and reflection spectra are shown in Fig. 4(b) by solid lines. Then, inserting the previously calculated effective wave parameters (see Fig. (3) into Eqs. (10) and (11), we obtain the Fresnel coefficients for an interface between the metamaterial and the host dielectric for waves propagating at $\theta=45 \mathrm{deg}$ and $\theta=135 \mathrm{deg}$. The transmission and reflection by a homogeneous slab characterized by these parameters and having the same five-layer thickness is then obtained by using these Fresnel coefficients in the standard equations for a Fabry-Perot etalon (see, e.g., Eqs. (25) and (26) in Ref. 11). The intensity transmittance and reflectance calculated in this way are shown by stars in Fig. 1(b). The obtained remarkable agreement between the analytical results and the direct numerical calculations confirms the homogenizability of the metamaterial and the correctness of our calculation approach.

\subsection{Influence of Metamolecular Orientation}

The influence of the dimer orientation can be analyzed in more detail by calculating the wave parameters as a function of the angle $\alpha$ at a fixed propagation direction, e.g., for $\theta=0$ [see Fig. 5(a)]. The incident light is, as before, assumed to be polarized along the $x$ axis to ensure that, for all choices of $\alpha$, the incident field points in the same direction relative to the discs, keeping the effect of optical anisotropy fixed. For the calculations, we select five particular wavelengths: $\lambda_{0}=525 \mathrm{~nm}$ (blue-detuned from the resonances), $\lambda_{0}=580 \mathrm{~nm}$ (nearly on-resonance 
(a)

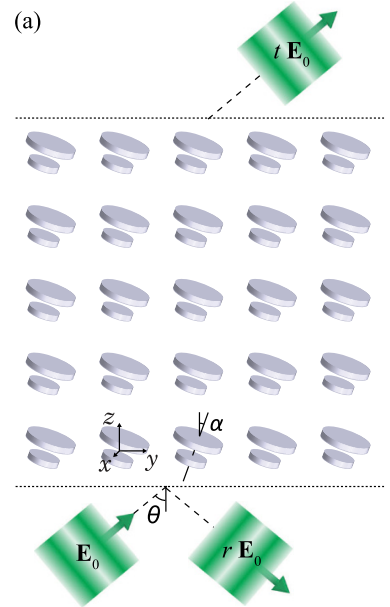

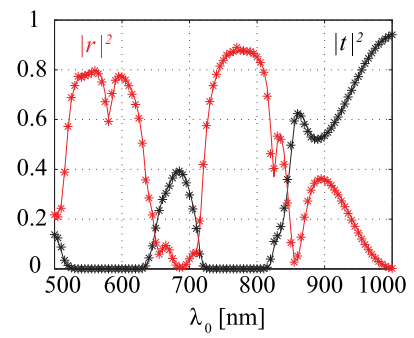

Fig. 4 (a) Transmission and reflection by a metamaterial slab composed of five planar arrays of metadimers. (b) Intensity transmittance and reflectance of the slab, when $\alpha=45$ deg and $\theta=45 \mathrm{deg}$. The medium surrounding the slab is the same as the medium surrounding the dimers. The solid lines are the result of direct numerical calculations, whereas the stars are calculated by using the approach of Sec. 目.

(a)

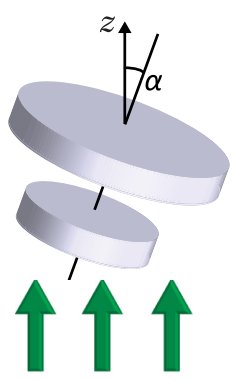

(c)

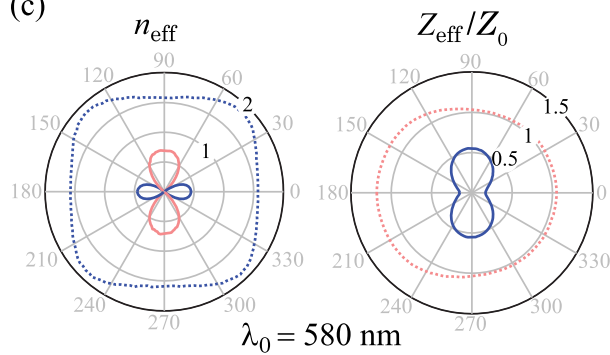

(e)

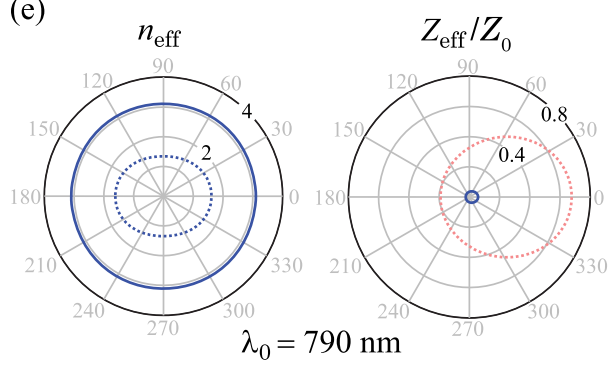

(b)

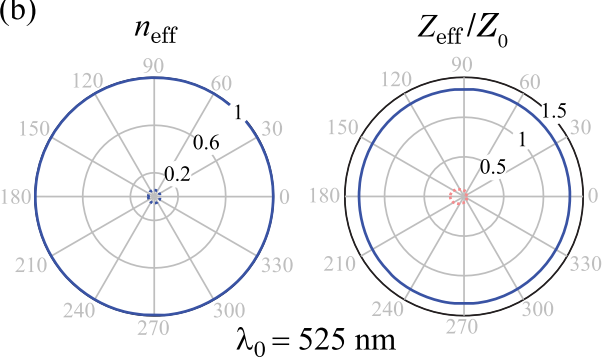

(d)

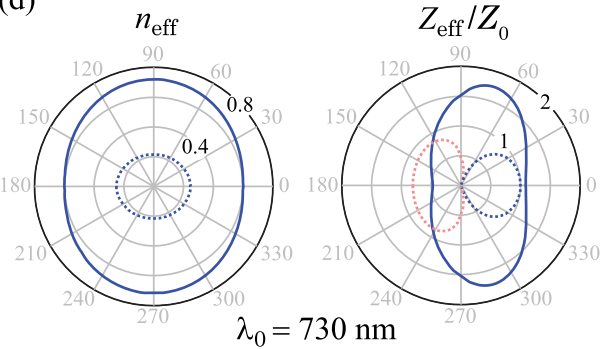

(f)

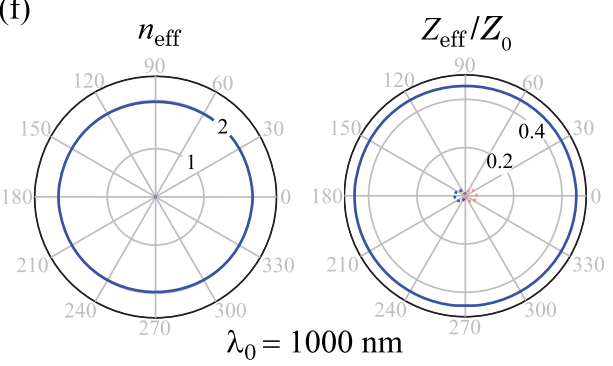

Fig. 5 The angle $\alpha$ between the orientation of the dimers and the $z$ axis is increased from 0 to $360 \mathrm{deg}$. The wave is TE-polarized and it propagates in the positive $z$-direction $(\theta=0 \mathrm{deg})$, as shown in (a). The calculated wave parameters, $n_{\text {eff }}$ and $Z_{\text {eff }} / Z_{0}$, are shown as functions of $\alpha$ for vacuum wavelengths of (b) $\lambda_{0}=525 \mathrm{~nm}$, (c) $\lambda_{0}=580 \mathrm{~nm}$, (d) $\lambda_{0}=730 \mathrm{~nm}$, (e) $\lambda_{0}=790 \mathrm{~nm}$, and (f) $\lambda_{0}=1000 \mathrm{~nm}$. The real and imaginary parts are shown by solid and dashed lines, respectively. The radius in the polar plots indicates the magnitude, whereas a dark blue (light red) color indicates whether the value is positive (negative). 
with the smaller disc), $\lambda_{0}=730 \mathrm{~nm}$ (between the two resonances), $\lambda_{0}=790 \mathrm{~nm}$ (nearly onresonance with the larger disc), and $\lambda_{0}=1000 \mathrm{~nm}$ (considerably red-detuned from the resonances).

For each of the selected wavelengths, we evaluate and present $n_{\text {eff }}$ and $Z_{\text {eff }}$ as functions of $\alpha$ in the polar plots of Fig. [ [ [Figs. 5(b) to [(f)] . Considering first the case of $\lambda_{0}=1000 \mathrm{~nm}$, shown in Fig. $[(\mathrm{f})$, we see that the wave parameters are independent of $\alpha$. At this long wavelength, the material responds like a spatially nondispersive dielectric with a refractive index of 2 . Similarly, the response at $\lambda_{0}=525 \mathrm{~nm}$, shown in Fig. 5(b), is nearly independent of $\alpha$. At this wavelength, the excitation in the dimers is essentially out-of-phase with the incident field, leading to a refractive index of $n_{\text {eff }}=1$. This is the value of the refractive index of vacuum. However, the material is not quite impedance-matched to vacuum. This means that, while optical refraction is absent, some reflection from the material will still take place.

The situation changes dramatically when the wavelengths approaching the dipole resonances of the discs are considered. For $\lambda_{0}=580 \mathrm{~nm}$, shown in Fig. 5(c), both wave parameters significantly depend on $\alpha$ and have large imaginary parts. The real part of the effective refractive index is observed to be negative when the dimer axis is about perpendicular $(90 \pm 50 \mathrm{deg})$ to the propagation direction of light (see the light red line). In contrast, at the resonance wavelength of the larger disc, $\lambda_{0}=790 \mathrm{~nm}$ [see Fig. [5(e)], the real part of the refractive index is positive and is quite large $\left(n_{\mathrm{eff}} \approx 3\right)$. At this wavelength, however, the wave impedance is essentially imaginary, leading to a high reflectivity. Finally, we consider the excitation at $\lambda_{0}=730 \mathrm{~nm}$ [see Fig. 5(d)]. At this wavelength, the imaginary parts of the wave parameters are smaller, and at certain values of $\alpha$, light can penetrate relatively deeply into the material. The refractive index and, especially, the impedance are found to depend strongly on $\alpha$. At $\alpha=75 \mathrm{deg}$, the imaginary part of $Z_{\text {eff }}$ crosses zero and becomes negative (see the light red dotted line). Note that for all five wavelengths, the conditions $\operatorname{Im}\left\{n_{\text {eff }}\right\}>0$ and $\operatorname{Re}\left\{Z_{\text {eff }}\right\}>0$ hold for all values of $\alpha$, as they should. From the obtained results, it is clear that the orientation of the dimers significantly affects the wave parameters when light is close to one of the resonance frequencies of the discs. We emphasize that this phenomenon has nothing to do with optical anisotropy and, therefore, can be considered as an additional tool for adjusting the properties of artificial optical materials.

\subsection{Influence of Light Propagation Direction and Polarization}

The relative orientation between the light propagation direction and the direction of the metamolecular alignment does not uniquely determine the effective wave parameters, because the orientation of the lattice is also important. Consequently, the wave parameters depend on the propagation direction $\theta$ in a different way than on the metamolecular orientation $\alpha$ considered in the previous subsection. Let us twist the metamolecules in the crystal cells to an angle $\alpha=$ $45 \mathrm{deg}$ and calculate $n_{\text {eff }}$ and $Z_{\text {eff }}$ as functions of $\theta$ at the five spectral locations selected previously. We first consider the TE-polarized light (polarized along the $x$ axis) that keeps the influence of optical anisotropy constant. The obtained wave parameters are shown in the polar plots of Fig. 6. The incidence angles close to $\pm 90 \mathrm{deg}$, at which the wave propagates along the surface, are excluded from the calculations (see the gray sectors) to avoid unrealistic numerical solutions in the form of abrupt jumps in the values of $n_{\text {eff }}$ and $Z_{\text {eff }}$. Far to the red from the resonances, at $\lambda_{0}=1000 \mathrm{~nm}$ [see Fig. 6(f)], the wave parameters are, as expected, independent of $\theta$. Also, at $\lambda_{0}=525 \mathrm{~nm}$ [Fig. 6(b)], the refractive index is independent of $\theta$. However, at this wavelength, we have $n_{\text {eff }} \approx 1$ and, therefore, any beam of light will propagate inside the material approximately as in vacuum [note the similarity with Fig. 5(b)]. The wave impedance is also seen to depend on $\theta$, but quite weakly, referring to an insignificant spatial dispersion in the metamaterial. The situation changes near the resonance wavelengths of the individual discs. At $\lambda_{0}=$ $580 \mathrm{~nm}$ [Fig. 6(c)] , the wave parameters are extremely sensitive to $\theta$. Here, the real part of the refractive index is close to zero when light propagates along the dimer axis $\theta=45$ deg or $\theta=225 \mathrm{deg}$ ), and then, at $\theta \approx-30 \mathrm{deg}$ or $\theta \approx 150 \mathrm{deg}$, it changes abruptly to the value of $\sim 2$ and stays constant within a range spanning $\sim 60 \mathrm{deg}$. Also, the wave impedance is extremely sensitive to $\theta$ at this wavelength. However, the large imaginary part of $Z_{\text {eff }}$ makes the metamaterial highly reflective when the material is surrounded by an ordinary dielectric medium. The same also holds around the second resonance, at $\lambda_{0}=790 \mathrm{~nm}$, where 
(a)

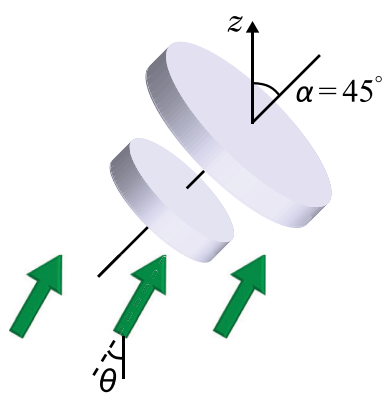

(c) $\quad n_{\text {eff }} \quad Z_{\text {eff }} / Z_{0}$

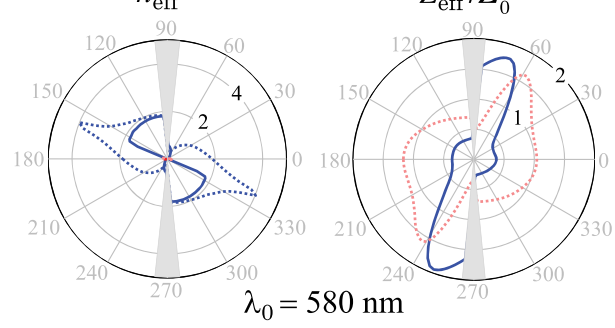

(e)

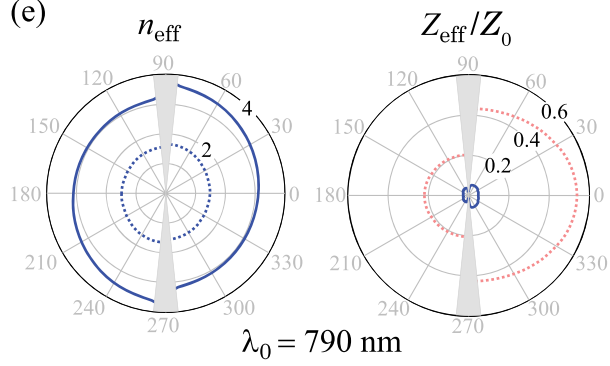

(b)

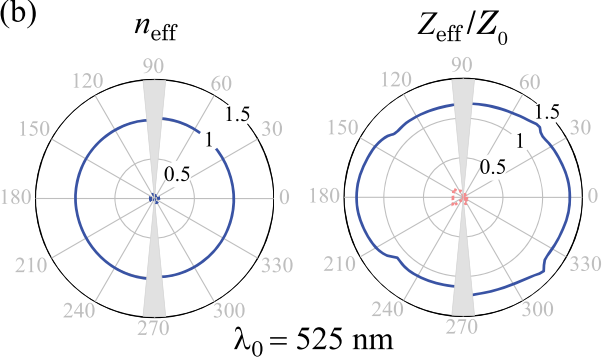

(d)

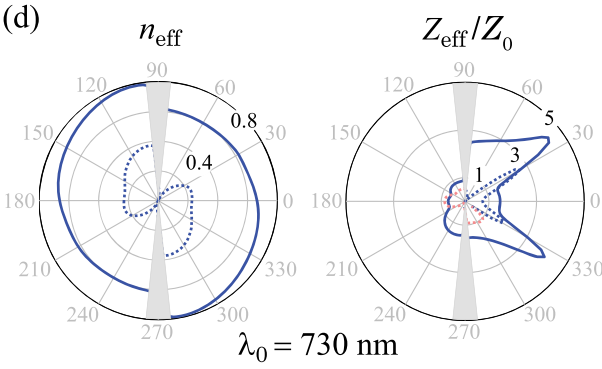

(f)

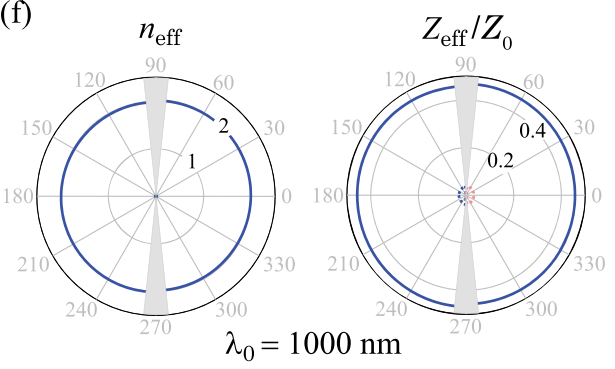

Fig. 6 The wave propagation direction in the host medium $\theta$ is changed from 0 to $360 \mathrm{deg}$. The wave is TE-polarized. The orientation of the dimers is fixed to $\alpha=45 \mathrm{deg}$, as shown in (a). The calculated wave parameters, $n_{\text {eff }}$ and $Z_{\text {eff }} / Z_{0}$, are shown as functions of $\theta$ for vacuum wavelengths of (b) $\lambda_{0}=525 \mathrm{~nm}$, (c) $\lambda_{0}=580 \mathrm{~nm}$, (d) $\lambda_{0}=730 \mathrm{~nm}$, (e) $\lambda_{0}=790 \mathrm{~nm}$, and (f) $\lambda_{0}=1000 \mathrm{~nm}$. The real and imaginary parts are shown by solid and dashed lines, respectively. The radius in the polar plots indicates the magnitude, whereas a dark blue (light red) color indicates whether the value is positive (negative).

$\operatorname{Im}\left\{Z_{\text {eff }}\right\} \gg \operatorname{Re}\left\{Z_{\text {eff }}\right\}$ [see Fig. 6e]]. The large refractive index at $\lambda_{0}=790 \mathrm{~nm}$ varies only slightly with $\theta$, which could be anticipated from the locally flat spectra shown earlier in Figs. B(a) and 3(b). Between the resonances, at the wavelength of $\lambda_{0}=730 \mathrm{~nm}$ [Fig. 6(d)], the wave impedance is large, almost real-valued, and dramatically depends on $\theta$.

It is obvious that the dependence of both the real and imaginary parts of $n_{\text {eff }}$ and $Z_{\text {eff }}$ on $\theta$ considerably differs from the dependence of these quantities on $\alpha$ obtained in the previous subsection. This means that the optical response of the metamaterial at a particular incidence angle $\theta$ can be efficiently tuned by the metamolecular alignment. The twist, indeed, makes the response strongly depend on the propagation direction via the phenomenon of spatial dispersion.

Let us next consider the TM polarization, for which the material exhibits optical anisotropy. Figure 7 shows the wave parameters obtained for this polarization using the same calculation method as before. The interplay between spatial dispersion and optical anisotropy is seen to provide further possibilities for adjusting the optical properties of metamaterials. At $\lambda_{0}=525 \mathrm{~nm}$ [Fig. 7(b)], the polar curve of the refractive index is not circular and significantly depends on $\theta$. Starting with $\theta \approx 50 \mathrm{deg}$, its real part changes quite abruptly, to a negative value. Also, the impedance at this angle has a sharp local maximum of its imaginary part and sharp features of the real part. At $\theta>40 \mathrm{deg}$, however, the waves will be essentially fully reflected from the material's surface and, therefore, the material is not a very promising negative-index material in this range. A different interesting range of incidence angles is found around $\theta=15 \mathrm{deg}$. In this range, the real part of $n_{\text {eff }}$ is close to 1 , and it shows a nearly flat polar curve. Simultaneously, the imaginary part of $n_{\text {eff }}$ is very small, and the impedance is essentially 


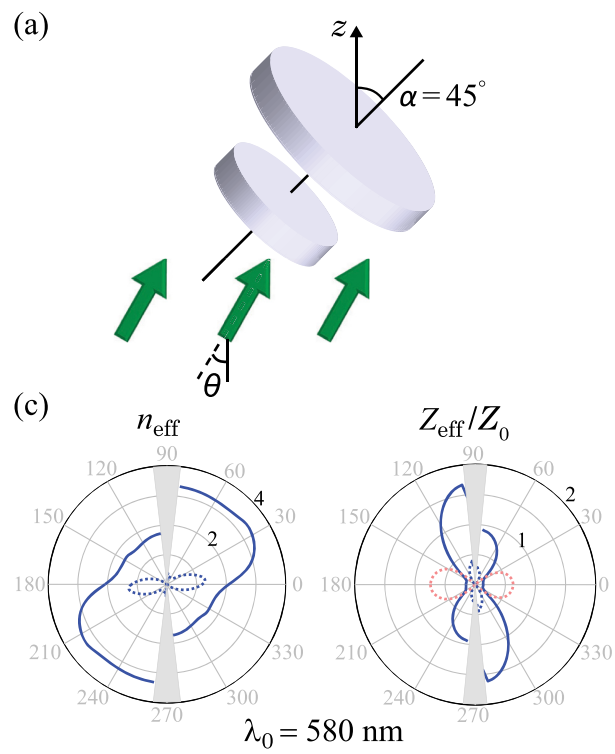

(e)

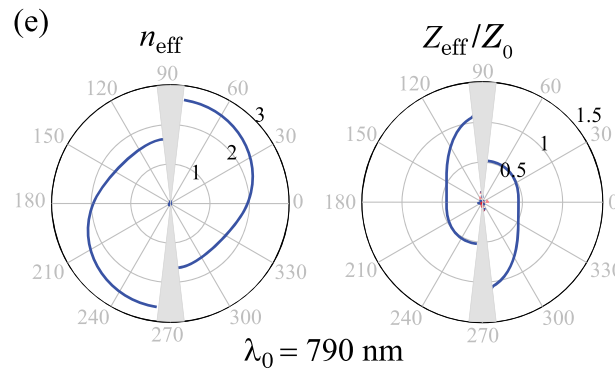

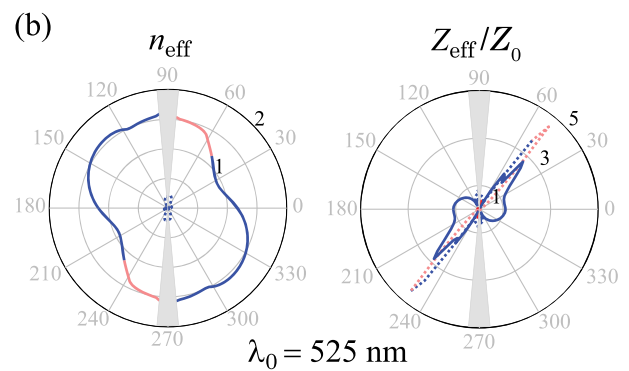

(d)

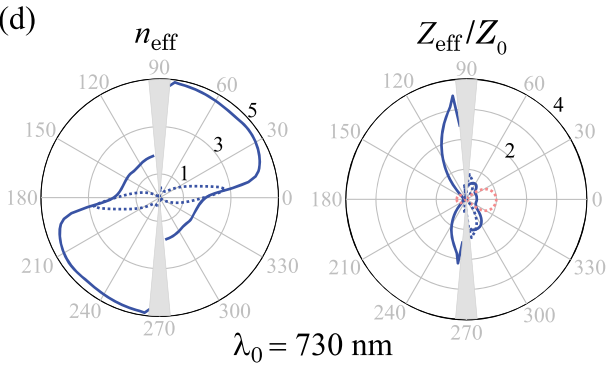

(f)

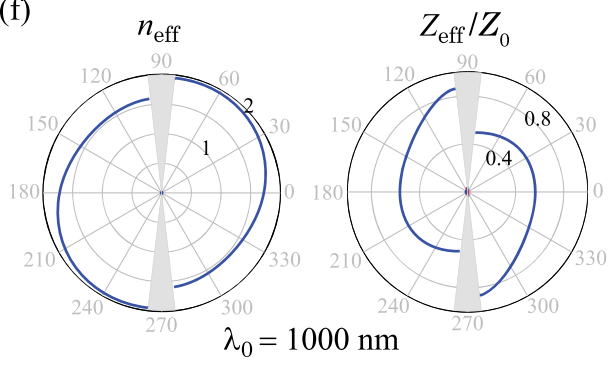

Fig. 7 The wave propagation direction in the host medium $\theta$ is changed from 0 to 360 deg. The wave is TM-polarized. The orientation of the dimers is fixed to $\alpha=45 \mathrm{deg}$, as shown in (a). The calculated wave parameters, $n_{\text {eff }}$ and $Z_{\text {eff }} / Z_{0}$, are shown as functions of $\theta$ for vacuum wavelengths of (b) $\lambda_{0}=525 \mathrm{~nm}$, (c) $\lambda_{0}=580 \mathrm{~nm}$, (d) $\lambda_{0}=730 \mathrm{~nm}$, (e) $\lambda_{0}=790 \mathrm{~nm}$, and (f) $\lambda_{0}=$ $1000 \mathrm{~nm}$. The real and imaginary parts are shown by solid and dashed lines, respectively. The radius in the polar plots indicates the magnitude, whereas a dark blue (light red) color indicates whether the value is positive (negative).

equal to that of vacuum. These conditions imply the possibility of achieving the self-collimation effect $\mathbb{Q}^{\mathrm{Q}}$ for a focused optical beam with a numerical aperture as high as 0.15 at reduced reflection and absorption losses. The beam can be incident onto the material from air at an angle of $\sim 23 \mathrm{deg}$. In the material, the beam will propagate at a larger angle (that is determined by the normal to the contour of $n_{\text {eff }}$ ) with a substantially reduced divergence.

Also, at $\lambda=1000 \mathrm{~nm}$ [see Fig. 7(f)], the contours of $n_{\text {eff }}$ and $Z_{\text {eff }}$ are not circular due to the influence of the optical anisotropy of the scatterers. The refractive index is, as expected, higher at positive values of $\theta$ than at negative values, due to the alignment of the dimers. Approximately the same behavior of $n_{\text {eff }}$ and $Z_{\text {eff }}$ is observed at $\lambda=790 \mathrm{~nm}$ [Fig. D(e)]. However, a quite flat contour of $n_{\text {eff }}$ is now observed at angles $\theta$ between -30 and $-60 \mathrm{deg}$. The real part of the refractive index in this region is close to that of glass and the imaginary part is quite small. Also, the impedance does not deviate much from its value in glass. Therefore, a focused beam incident onto the material from glass at $\theta \approx 45 \mathrm{deg}$ can be expected to show self-collimation with low reflection and absorption losses. Its numerical aperture can, in this case, be as high as 0.25 . We emphasize that the parameter values and the geometry of the metamaterial units were not specially optimized to obtain self-collimation.

At $\lambda=580 \mathrm{~nm}$ [Fig. 7(c)] and $730 \mathrm{~nm}$ [Fig. 7(d)], optical anisotropy also plays a significant role, leading to a high value of the real part of $n_{\text {eff }}$ at positive values of $\theta$. At $\lambda=730 \mathrm{~nm}$, it nearly reaches the value of 5 . The material is clearly bifacial at these wavelengths, which can be seen from the noncentrosymmetric contours of $Z_{\text {eff }}$, especially in Fig. $7(\mathrm{~d})$. The reflection and absorption losses, however, are high in these two cases. 


\section{Summary}

In this work, we have introduced a concept of internally twisted achiral metamaterials and presented a general technique for evaluating their optical characteristics. Such materials are composed of noncentrosymmetric metamolecules that are tilted with respect to the lattice. In these propagationally anisotropic materials, both the effective refractive index $n_{\text {eff }}$ and wave impedance $Z_{\text {eff }}$ significantly depend on the propagation direction of light, even if the light polarization is such that the optical anisotropy does not contribute to this dependence. Thus, to characterize these materials, it is necessary to consider the wave parameters as functions of not only the frequency and polarization, but also the propagation direction of light. Previously used Fresnel transmission and reflection coefficients are not valid for such materials. We have, therefore, generalized these coefficients to allow for the incident and reflected waves to see different optical media.

The applicability of our calculation technique was demonstrated by investigating an optical metamaterial composed of paired silver discs embedded in glass. In the metamaterial, the common orientation of the dimers was considered to be independent of the orientation of the crystal lattice, which provided an additional control over the spatial dispersion of the material. For this metamaterial, we have verified that the introduced wave parameters do not depend on the number of molecular layers in the material and are, therefore, defined unambiguously. We have studied in detail the dependence of $n_{\text {eff }}$ and $Z_{\text {eff }}$ on the orientation of the metamolecules and found that, close to the resonance wavelengths of the discs, both the refractive index and the wave impedance are significantly influenced by this orientation. We have also analyzed the dependence of $n_{\text {eff }}$ and $Z_{\text {eff }}$ on the propagation direction of light. This dependence was shown to give a powerful tool for adjusting the metamaterial properties in addition to classical optical anisotropy. This fact opens up new opportunities for designing functional metamaterials which are purposefully tailored to reflect, refract, or guide light beams by harnessing the dependence of their plane-wave components on the propagation direction. As an example, we have demonstrated the possibility of achieving low-loss self-collimation for optical beams by making use of spatial dispersion and optical anisotropy in an optical metamaterial. Compared to photonic crystals, metamaterials can be designed to provide equal reflection coefficients for the plane-wave components of a focused beam by adjusting the values of the wave impedance within the range of the relevant incidence angles. This would provide diffraction-free guiding of optical beams without altering their angular spectra.

\section{Acknowledgments}

We acknowledge financial support from the Academy of Finland and publication of a conference paper ${ }^{17}$ preceding the present article by SPIE.

\section{References}

1. A. Sihvola, Electromagnetic Mixing Formulas and Applications, IEEE, London (1999).

2. G. W. Milton, The Theory of Composites, Cambridge University Press, Cambridge (2002).

3. A. I. Căbuz, D. Felbacq, and D. Cassagne, "Spatial dispersion in negative-index composite metamaterials," Phys. Rev. A 77, 013807 (2008).

4. B. Gompf et al., "k-dependent optics of nanostructures: spatial dispersion of metallic nanorings and split-ring resonators," Phys. Rev. B 86, 075462 (2012).

5. H. Kosaka et al., "Super-prism phenomena in photonic crystals: toward microscale lightwave circuits," J. Lightwave Technol. 17(11), 2032-2038 (1999).

6. H. Kosaka et al., "Photonic crystals for micro lightwave circuits using wavelength-dependent angular beam steering," Appl. Phys. Lett. 74(10), 1370-1372 (1999).

7. J. Arlandis et al., "Mesoscopic self-collimation and slow light in all-positive index layered photonic crystals," Phys. Rev. Lett. 108, 037401 (2012).

8. Z. Lu et al., "Experimental demonstration of self-collimation inside a three-dimensional photonic crystal," Phys. Rev. Lett. 96, 173902 (2006). 
9. P. T. Rakich et al., "Achieving centimetre-scale supercollimation in a large-area two-dimensional photonic crystal," Nat. Mater. 5, 93-96 (2006).

10. C. Luo et al., "All-angle negative refraction without negative effective index," Phys. Rev. B 65, 201104 (2002).

11. P. Grahn, A. Shevchenko, and M. Kaivola, "Theoretical description of bifacial optical nanomaterials," Opt. Express 21(20), 23471-23485 (2013).

12. P. Grahn, A. Shevchenko, and M. Kaivola, "Interferometric description of optical metamaterials," New J. Phys. 15(11), 113044 (2013).

13. P. Grahn, A. Shevchenko, and M. Kaivola, "Electric dipole-free interaction of visible light with pairs of subwavelength-size silver particles," Phys. Rev. B 86, 035419 (2012).

14. P. Grahn, A. Shevchenko, and M. Kaivola, "Multipole polarizability of a nanodimer in optical waves," t. Eur. Opt. Soc. 8, 13009 (2013).

15. P. Grahn, A. Shevchenko, and M. Kaivola, "Electromagnetic multipole theory for optical nanomaterials," New J. Phys. 14(9), 093033 (2012).

16. P. B. Johnson and R. W. Christy, "Optical constants of the noble metals," Phys. Rev. B 6, 4370-4379 (1972).

17. P. Grahn, A. Shevchenko, and M. Kaivola, "Internally twisted non-centrosymmetric optical metamaterials," Proc. SPIE 9125, 91251L (2014).

Andriy Shevchenko received his DSc (Tech.) degree in physics from Helsinki University of Technology in 2004. In 2007, he was appointed as docent in optical physics, and since 2012, he has held the position of university lecturer at Aalto University, Finland. He is the author of more than 100 scientific publications in international journals and conference proceedings. His research interests include theory of light-matter interaction, nano-optics, statistical optics, optical nanomaterials, and methods of micro- and nanofabrication.

Patrick Grahn received his DSc (Tech.) degree in engineering physics from Aalto University, School of Science, in 2014. His research interests include computational electromagnetism, novel optical technologies, metamaterials, and simulations.

Matti Kaivola is a full professor in optical physics and the head of the Department of Applied Physics at Aalto University, Finland. He is the author of more than 200 scientific publications in international journals and conference proceedings. He leads the research of the Optics and Photonics group at Aalto University on the topics of electromagnetic optics, nanophotonics, laser physics and microacoustics. 